\title{
Third Engel groups and the Macdonald-Neumann conjecture
}

\author{
S. Bachmuth and H.Y. Mochizuki
}

\begin{abstract}
There exists a non-solvable group which is third Engel. More generally, the existence of a non-solvable group in which every $n$-generator subgroup is nilpotent of class at most $2 n-1$ is confirmed.
\end{abstract}

\section{Introduction}

$G$ is an Engel group if for any elements $x, y$ in $G$, the commutator $[x, y, y, \ldots, y]=1$ where $y$ is repeated $n(x, y)$ times and $n(x, y)$ may depend on $x$ and $y$. If $n=n(x, y)$ is independent of $x$ and $y$, then $G$ is said to be a bounded Engel group of degree $n$ or simply an $n$-th Engel group.

The first example of a non-solvable Engel group was a consequence of the work of Golod-Safarevič [2]. (See the example in Herstein [7], p. 124.) The group constructed in Section 4 of [1] is the first example of a nonsolvable bounded Engel group, although the exact value of the degree $n$ was not determined in [1]. This value has now been determined to be three, and reasons for its interest will now be advanced.

In the above notation, if $n=1, G$ is abelian, and if $n=2, G$ is metabelian. Also well-known is the fact that bounded Engel 2-groups need not be nilpotent. However, Gupta in [3] has shown that third Engel 2-groups are solvable. His work is based on results of Gupta and Weston [4] on groups of exponent 4 and results of Heineken [5] on third Engel groups, and he actually shows that a third Engel group is the extension of

Received 5 July 1971. This research was supported by a grant from the National Science Foundation of the USA. 
a solvable group by a group of exponent 5. Thus, the problem of whether third Engel groups are solvable reduces to the corresponding question for 5-groups. The existence of such a nonsolvable group had already been conjectured by Macdonald and Neumann in [10], p. 557. We show

THEOREM 1. There exists a third Engel group of exponent 5 which is not solvable.

COROLLARY 1. There exists a nonsolvable group all of whose two generator subgroups are nilpotent of class (at most) three.

COROLLARY 2. The variety of 2-metabelian groups (that is, each two generator subgroup is metabelian) is a nonsolvable variety.

The Macdonald-Neumann conjecture is that there exist [2 +3$]$ 5-groups of nilpotency class $m$ for each $m \geq 1$. An $[n \rightarrow k]$ group is a group such that every $n$-generator subgroup is nilpotent of class $\leq k$. Theorem $l$ is equivalent to the Macdonald-Neumann conjecture as pointed out by Gupta in [3]. Direct verification of the conjecture readily follows by using Zusatz 2 of Heineken [6] which states that the group of Theorem 1 is automatically a [2 $\rightarrow 3]$ group. Hence the Corollary 1 . In relation to the group of Theorem 1, we have in fact the more general

THEOREM 2. A third Engel group of exponent 5 is an $[n \rightarrow(2 n-1)]$ group. In particular the group of Theorem 1 is a nonsolvable [ $\rightarrow(2 n-1)]$ group.

Gupta has shown that groups of type $[n \rightarrow(2 n-2)]$ are nilpotent by abelian and hence solvable. (In fact, according to Neumann [11], p. 98, Heineken has shown that groups of this type without elements of order 2 are nilpotent of class at most $3 n-3$.$) Furthermore, Gupta has shown$ that $[n \rightarrow(2 n-1)]$ groups are extensions of a solvable group by a group of exponent 5 . Thus Theorem 2 points out where the dichotomy occurs between solvability and nonsolvability for groups of type $[n \rightarrow k]$.

\section{Some required notation and results}

Let $R$ be the free associative noncommutative ring of characteristic 5 with identity generated by indeterminates $x_{1}, x_{2}, x_{3}, \ldots$. Let $L$ be the Lie ring in $R$ generated by the $x_{i}$ where addition in $L$ is the same as in $R$ and Lie multiplication in $L$ is commutation $[x, y]=x y-y x$. 
Let $H$ denote the ideal of $R$ generated by all elements

$$
g(x, y)=x^{2} y-3 x y x+3 y x^{2},
$$

where $x, y$ are any elements of $L$, or, alternately, by all elements

$$
h(x, y, z)=x y z+z y x+2 y z x+2 y x z,
$$

where $x, y, z$ are arbitrary elements of $L . H$ is a Lie substitution ideal, which means that if a polynomial $P$ in the $x_{i}$ is in $H$, so is the polynomial $P^{\prime}$ obtained from $P$ by substituting elements of $L$ for the $x_{i}$. Let $U$ be the ideal of $R$ generated by all monomials in the $x_{i}$ with a repeated indeterminate factor. We state the main result of [1].

THEOREM A. In $R /(H+U)$, let $G$ be the group generated by the $\left(1+x_{i}\right) \bmod (H+U)$. Then $G$ is a nonsolvable group of exponent 5 .

In the next section, we will show that $G$ is third Engel.

Let $y_{1}, y_{2}, \ldots, y_{n}$ be distinct indeterminates, $n \geq 3$, and let $T_{3}\left(y_{1}, y_{2}, \ldots, y_{n}\right)$ be the multilinear (degree 1 in each $y_{i}$ ) part of

$$
\left[\left(1+y_{1}\right)\left(1+y_{2}\right) \ldots\left(1+y_{n}\right)-1\right]^{3}
$$

It readily follows that

(1) $T_{3}\left(\ldots, y_{i}, y_{i+1}, \ldots\right)$

$$
=T_{3}\left(\ldots, y_{i+1}, y_{i}, \ldots\right)+T_{3}\left(\ldots,\left[y_{i}, y_{i+1}\right], \ldots\right) \text {. }
$$

If $T$ is the ideal of $R$ generated by the $T_{3}\left(z_{1}, \ldots, z_{n}\right), n \geq 3$, where the $z_{i}$ are in $L$, then $T \subseteq B$ (Lemma 4 of [1]). As a result (see Section 4 of [1]), we have $(g-1)^{3}=0$ for all $g \in G$.

We will also need the following three theorems.

THEOREM B. In $R$

(i) a monomial of the form $M_{1} x_{i} M_{2} x_{i} M_{3}$ is congruent to $\alpha P x_{i}^{2}$ $\bmod H$, for all indeterminates $x_{i}$, where $P$ is a polynomial in the $x_{j}$ and $\alpha$ is an integer moduzo 5 , 
(ii) modulo $H, x_{i}^{2}$ commutes with alz elements of $R$, and

(iii) if a monomial $M$ in the $x_{i}$ has an indeterminate factor repeated three or more times, then $M \equiv 0 \bmod H$.

THEOREM C (Heineken, Hauptsatz 3 of [5]). In a group without elements of even order, the set of alz elements $g$ satisfying $\left[g^{ \pm 1}, y, y, y\right]=1$ for all $y$ in the group forms a subgroup.

THEOREM D. The associated lie ring of a third Engel group of exponent 5 is a third Engel Lie ring of characteristic 5 .

All parts of Theorem B are trivial consequences of the results or methods in Section 3 of [1]. A proof of Theorem D readily follows from the same arguments used in Theorem 4 of Higman [9]. Theorem $\mathrm{C}$ is proved by Heineken using right-normed notation. But as shown by Lemma $I$ of [10], the third Engel conditions in the right-normed and the left-normed notations are equivalent, that is,

$$
\begin{aligned}
& {[y,[y,[y, x]]]=1 \text { if and only if }[x, y, y, y]=1 \text { for }} \\
& \text { elements } x \text { and } y \text { in a group } G \text {. }
\end{aligned}
$$

\section{Proof of Theorem 7}

Let $G$ be the group of Theorem A in Section 2. To prove Theorem 1 we need to show that $G$ is a third Engel group. By Theorem $C$, we need to prove that $[g, y, y, y]=1$ for all $y \in G$ where $g$ is a generator of $G$. Put $g=1+x$ and $y=1+P$ where $x$ is an indeterminate (generator of $R$ ) and $P$ is a polynomial in the $x_{i}$.

LEMMA 1. $[g, y, y, y] \equiv 1+P x P^{2}+P^{2} x P \bmod (H+U)$.

Proof. $g^{-1}=1-x$ and $y^{-1}=1-P+P^{2}$ modulo $(H+U)$, since $x^{2} \equiv P^{3} \equiv 0 \bmod (H+U)$. Thus, all terms omitted from $[g, y, y, y]$ either have two occurrences of $x$ or have a factor of $P^{3}$.

LEMMA 2. $P x P^{2} \equiv P^{2} x P \equiv 0 \bmod (H+U)$.

Proof. Modulo $U, P x P^{2}$ is the sum of polynomials $T_{3}^{*}\left(x_{i_{1}}, x_{i_{2}}, \ldots, x_{i_{k}}\right)$ where the $x^{\prime} \mathrm{s}$ are indeterminates and 
$T_{3}^{*}\left(x_{1}, x_{2}, \ldots, x_{k}\right)$ is the sum of monomials $M_{1} x M_{2} M_{3}, M_{i}$ coming from the $i$-th factor of $P$ and $M_{1} M_{2} M_{3}$ having degree one in $x_{i}$, $1 \leq i \leq k$. Note that the sum of the monomials $M_{1} M_{2} M_{3}$ is $T_{3}\left(x_{1}, x_{2}, \ldots, x_{k}\right)$. We need only prove that

$T_{3}^{*}\left(x_{1}, x_{2}, \ldots, x_{k}\right) \equiv 0 \bmod H$ for $k \geq 3$. We proceed by induction on $k$. For $k=3$, we have

$T_{3}^{*}\left(x_{1}, x_{2}, x_{3}\right)=x_{1} x_{2} x_{3}+x_{1} x x_{3} x_{2}+x_{2} x x_{1} x_{3}+x_{2} x x_{3} x_{1}+$ $x_{3} x x_{1} x_{2}+x_{3} x x_{2} x_{1}$

But the sum of these six monomials is in $H$ because it is just the linearization of $x_{1} x x_{1} x_{1}$ which is certainly in $H$ from part (ii) or (iii) of Theorem B. Therefore, assume inductively that $T_{3}^{*}\left(x_{1}, x_{2}, \ldots, x_{k}\right) \equiv 0 \bmod H$. Since $H$ is a substitution ideal, $T_{3}^{*}\left(z_{1}, z_{2}, \ldots, z_{k}\right) \equiv 0 \bmod H$ with $z_{i} \in L$. We need the equation

(2) $T_{3}^{*}\left(x_{1}, \ldots, x_{i}, x_{i+1}, \ldots, x_{k+1}\right)-T_{3}^{*}\left(x_{1}, \ldots, x_{i+1}, x_{i}, \ldots, x_{k+1}\right)$

$$
=T_{3}^{*}\left(x_{1}, \ldots, x_{i} x_{i+1}-x_{i+1} x_{i}, \ldots, x_{k+1}\right) \text {. }
$$

(The proof of (2) is essentially the same as that for (1).)

Notice that the expression on the right-hand side of (2) has fewer arguments and so by our induction hypothesis is in $H$. Using this, we have

$$
\begin{aligned}
T_{3}^{*}\left(x_{1}, x_{2},\right. & \left.x_{3}, \ldots, x_{k+1}\right) \\
= & 6 T_{3}^{*}\left(x_{1}, x_{2}, x_{3}, \ldots, x_{k+1}\right) \\
\equiv & T_{3}^{*}\left(x_{1}, x_{2}, x_{3}, \ldots, x_{k+1}\right)+T_{3}^{*}\left(x_{1}, x_{3}, x_{2}, \ldots, x_{k+1}\right) \\
& +T_{3}^{*}\left(x_{2}, x_{1}, x_{3}, \ldots, x_{k+1}\right)+T_{3}^{*}\left(x_{2}, x_{3}, x_{1}, \ldots, x_{k+1}\right) \\
& +T_{3}^{*}\left(x_{3}, x_{1}, x_{2}, \ldots, x_{k+1}\right)+T_{3}^{*}\left(x_{3}, x_{2}, x_{1}, \ldots, x_{k+1}\right) \bmod H .
\end{aligned}
$$

But again the six terms on the right-hand side are $\equiv 0 \bmod H$, since they are the linearization of

$$
T_{3}^{*}\left(x, x, x, x_{4}, \ldots, x_{k+1}\right) \equiv 0 \bmod H .
$$

This completes the induction. By symmetry $P^{2} x P \equiv 0 \bmod H$. This proves 
Lemma 2 and hence Theorem 1.

\section{Proof of Theorem 2}

Let $G$ be the free third Engel n-generator group of exponent 5 . Theorem 1 and the results of Heineken and Gupta show that $G$ is nilpotent of class $\geq 2 n-1$. To show that $G$ has class $\leq 2 n-1$, it is sufficient to prove that the associated Lie ring of $G$ is nilpotent of class $\leq 2 n-1$. As a result of Theorem $D$ we need to prove

LEMMA 3. The free third Engel n-generator Lie ring $L$ of characteristic 5 is nilpotent of class $\leq(2 n-1)$.

Proof. Let $a_{1}, a_{2}, \ldots, a_{n}$ generate $L$, and let $R$ be the associative subring of the endomorphism ring of $(L,+)$ generated by the endomorphisms

$$
A_{i}=\operatorname{ada} a_{i} x \rightarrow\left[x, a_{i}\right]
$$

together with the identity map. According to Higgins [8], $R$ is a homomorphic image of the ring $R / H$ of Section 2 .

To prove that $L$ is nilpotent of class $\leq 2 n-1$, it is sufficient to prove that every left-normed product

$$
c=\left[a_{i_{1}}, a_{i_{2}}, a_{i_{3}}, \ldots, a_{i_{2 n}}\right]=\left[\ldots\left[\left[a_{i_{1}}, a_{i_{2}}\right], a_{i_{3}}\right], \ldots, a_{i_{2 n}}\right]=0 .
$$

We consider three cases:

(i) Some $a_{i j}, j \geq 2$, appears at least three times in $c$. Then

$$
\begin{aligned}
c & =a_{i_{1}} A_{i_{2}} \cdots A_{i_{2 n}} \\
& =0 \text { by }(i i i) \text { of Theorem } B .
\end{aligned}
$$

(ii) $a_{i_{1}}$ appears at least three times in $c$. Then

$$
\begin{aligned}
c & =\alpha a_{i_{1}} A_{i_{1}}^{2} P \text { by }(i) \text { and }(i i) \text { of Theorem } B \\
& =0 .
\end{aligned}
$$

(iii) Each $a_{i}$ appears twice in $c$. Then 


$$
\begin{aligned}
c & =\alpha a_{i_{1}}{ }^{A}{ }_{1}{ }^{M} \\
& =0
\end{aligned}
$$

where $M$ is the product of the squares of the other indeterminates (by (i) and (ii) of Theorem B).

This exhausts all cases and completes the proof of Lemma 3 .

\section{References}

[1] S. Bachmuth, H.Y. Mochizuki, D.W. Walkup, "Construction of a nonsolvable group of exponent $5 "$, Word problems (edited by W.W. Boone, R.C. Lyndon, F.B. Cannonito. North-Holland, Amsterdam; in press).

[2] E.S. Golod, "On nil-algebras and residually finite p-groups" (Russian), Izv. Akad. Nauk SSSR Ser. Mat. 28 (1964), 273-276.

[3] N.D. Gupta, "Third Engel 2-groups are soluble", Canad. Math. Bulz. (to appear).

[4] Narain D. Gupta and Kenneth W. Weston, "On groups of exponent four", J. Algebra 17 (1971), 59-66.

[5] Hermann Heineken, "Engelsche Elemente der Länge drei", IZZinois $J$. Math. 5 (1961), 681-707.

[6] Hermann Heineken, "Über ein Levisches Nilpotenzkriterium", Arch. Math. 12 (1961), 176-178.

[7] I.N. Herstein, Topics in ring theory (Lecture notes, Univ. Chicago, Chicago, 1965).

[8] P.J. Higgins, "Lie rings satisfying the Engel condition", Proc. Cambridge Philos. Soc. 50 (1954), 8-15.

[9] Graham Higman, "On finite groups of exponent five", Proc. Cambridge Philos. Soc. 52 (1956), 381-390.

[10] I.D. Macdonald and B.H. Neumann, "A third-Engel 5-group", $J$. Austral. Math. Soc. 7 (1967), 555-569. 
[11] Hanna Neumann, Varieties of groups (Ergebnisse der Mathematik und ihrer Grenzgebiete, Band 37. Springer-Verlag, Berlin, Heidelberg, New York, 1967).

Department of Mathematics, University of California, Santa Barbara, California, USA. 